\title{
Dynamic genome-scale cell-specific metabolic models reveal novel inter-cellular and intra-cellular metabolic communications during ovarian follicle development
}

\author{
Beatriz Peñalver Bernabé1, Ines Thiele ${ }^{2}$, Eugene Galdones ${ }^{3}$, Anaar Siletz ${ }^{3}$, Sriram Chandrasekaran 4 , \\ Teresa K. Woodruff ${ }^{3,5}$, Linda J. Broadbelt ${ }^{6}$ and Lonnie D. Shea ${ }^{4^{*}}$ (D)
}

\begin{abstract}
Background: The maturation of the female germ cell, the oocyte, requires the synthesis and storing of all the necessary metabolites to support multiple divisions after fertilization. Oocyte maturation is only possible in the presence of surrounding, diverse, and changing layers of somatic cells. Our understanding of metabolic interactions between the oocyte and somatic cells has been limited due to dynamic nature of ovarian follicle development, thus warranting a systems approach.

Results: Here, we developed a genome-scale metabolic model of the mouse ovarian follicle. This model was constructed using an updated mouse general metabolic model (Mouse Recon 2) and contains several key ovarian follicle development metabolic pathways. We used this model to characterize the changes in the metabolism of each follicular cell type (i.e., oocyte, granulosa cells, including cumulus and mural cells), during ovarian follicle development in vivo. Using this model, we predicted major metabolic pathways that are differentially active across multiple follicle stages. We identified a set of possible secreted and consumed metabolites that could potentially serve as biomarkers for monitoring follicle development, as well as metabolites for addition to in vitro culture media that support the growth and maturation of primordial follicles.
\end{abstract}

Conclusions: Our systems approach to model follicle metabolism can guide future experimental studies to validate the model results and improve oocyte maturation approaches and support growth of primordial follicles in vitro.

Keywords: Ovarian follicle development, Metabolism, Metabolic communities, Secreted metabolites, Cell-type specific metabolic models, Genome-scale modeling

\section{Background}

Understanding the complex intercellular metabolic interactions during ovarian follicle development requires a systems biology approach. The follicle consists of somatic cells that surround the female germ cell, the oocyte. Metabolic communication between these cell types is

\footnotetext{
* Correspondence: Idshea@umich.edu

${ }^{4}$ Department of Biomedical Engineering, University of Michigan, Ann Arbor, MI 48109, USA

Full list of author information is available at the end of the article
}

necessary for follicle development and oocyte maturation. Yet, most systems-level studies of the follicle to date have focused on signaling and gene regulation [1] rather than on metabolism. While the metabolic interaction between granulosa cells and oocytes during development has been documented [2-5], a systems biology analysis provides a comprehensive perspective that is not possible using bottom-up methods measuring a few components at a time $[6,7]$. Current untargeted metabolomics methods are not feasible for studying ovarian follicle metabolism due to the large number of cells $(>10,000)$ that are required,

(c) The Author(s). 2019 Open Access This article is distributed under the terms of the Creative Commons Attribution 4.0 International License (http://creativecommons.org/licenses/by/4.0/), which permits unrestricted use, distribution, and reproduction in any medium, provided you give appropriate credit to the original author(s) and the source, provide a link to the Creative Commons license, and indicate if changes were made. The Creative Commons Public Domain Dedication waiver (http://creativecommons.org/publicdomain/zero/1.0/) applies to the data made available in this article, unless otherwise stated. 
specifically for oocyte isolation. One way to overcome this limitation is by applying a systems biology approach to model ovarian follicle metabolism using transcriptomics data. Systems biology approaches can reveal key secreted and consumed metabolites, and dynamic metabolic processes that occur during mouse folliculogenesis in the oocyte and somatic cells.

Here we apply genome-scale network models to model follicle metabolism [8]. These network models are manually curated and represent the relationship between genes, proteins and metabolites in a system. They have been successfully employed to study of the metabolism of unicellular and multicellular organisms [9], including mammals [10]. The metabolic network models for multicellular organisms contain all possible biochemical reactions that happen in an organism based on literature evidence. For example, the human network model by Thiele et al. contains 7440 reactions, 1789 genes, 2194 transcripts, 2657 proteins, 1052 protein complexes, and 5063 metabolites [11]. Transcriptomics, proteomics or metabolomics data can be integrated with genome-scale metabolic models to create context-specific or cell-type specific models that represent metabolic reactions that are active in a cell-type. Such context-specific models have been applied successfully to predict metabolic behaviors of human and mouse tissues [12-15].

To build our cell-type specific metabolic models, we used the mouse metabolic reconstruction [16], and updated it based on the more comprehensive human metabolic model [11]. Using this updated mouse metabolic reconstruction and transcriptomic data of ovarian follicle cells, we next built a cell-type specific mouse ovarian follicle metabolic reconstruction [17]. We then explored this model to identify the most active metabolic communities and pathways. We further identified secreted and consumed metabolites at each stage of mouse ovarian follicle development for each cell type (e.g., oocyte, cumulus granulosa cells). Our study provides insights on the communication and dependence of the multiple cells types that comprise the ovarian follicle. Secreted and consumed metabolites identified by this approach in the growing ovarian follicle can be used to improve in vitro follicle culture systems, and to develop novel biomarkers of oocyte quality for in vitro fertilization (IVF).

\section{Results}

\section{Updating the mouse general metabolic model}

A comprehensive mouse metabolic reconstruction based on the most up-to-date metabolic knowledge could increase the accuracy of a reconstruction. Mouse Recon 1 was unable of adequately modelling multiple mouse metabolic functions, several of them associated with key follicle metabolic pathways (e.g., the production of estrogen metabolites). Thus, we constructed a high quality and more comprehensive mouse metabolic reconstruction, called Mouse Recon 2, employing the current best practices in systems biology [11] (Additional files 1 and 2). Mouse Recon 2 combines the previous established Mouse Recon 1 [16] with the metabolic pathways that have human homologues in the human metabolic reconstruction, Human Recon 2 [11] and several key ovarian follicle development metabolic pathways that were not included in either of the two reconstructions (Additional file 9: Note S1 and Note S2). The new Mouse Recon 2 contained a total of 2082 new reactions and 754 new unique metabolites (Table 1). Out of these new reactions, 700 of them were catalyzed by 251 enzymes that were not previously included in Mouse Recon 1 . The genes that encode these new enzymes were highly enriched in oxidative phosphorylation processes and androstenedione and testosterone biosynthesis and metabolism (Additional files 8 and 9: Table S1).

Comparison of the metabolic pathways between Mouse Recon 2 and Mouse Recon 1 showed that 12 metabolic pathways were completely new, such as androgen and estrogen metabolism, arachidonic acid metabolism, and cytochrome metabolism. A total of 51 metabolic pathways were updated, some of these are known to be involved in ovarian follicle maturation, e.g., vitamin D, cholesterol, and steroid metabolism (Additional file 9: Figure S1). Additionally, we identified 43 genes in Mouse Recon 2 that have human homologues but were not included in Human Recon 2 (Additional file 9: Table S2). Finally, we checked the model metabolic functionality (Additional file 3). Out of 363 distinct metabolic functions (e.g., production of ATP from glucose), Mouse Recon 2 was able to successfully simulate $93 \%$ of the tested metabolic functions, while Mouse Recon 1 could only simulate $84 \%$ of those functions.

\section{Creation of a mouse ovarian follicle specific metabolic reconstruction}

Among the 6172 reactions in Mouse Recon2, only a small subset of reactions is likely to be active in follicle cells. We hence constructed an ovarian follicle specific metabolic model, OvoFol Recon 1 (Methods; Additional files 4 and 5). OvoFol Recon 1 was obtained by integrating four sets of mouse follicles transcriptomics data (Additional file 9: Table S3). This transcriptomics set includes our data from freshly isolated follicles and oocytes [18] across all stages of follicle development, cumulus cells and mural cells during in vivo follicle maturation [19], cumulus cells during the in vivo acquisition of oocyte competence [20] (Fig. 1a, b). OvoFol Recon 1 contained 3992 reactions, 1364 unique metabolites, and 1871 genes (Table 1). OvoFol Recon 1 has 2180 reactions fewer than Mouse Recon 
Table 1 Comparisons between Mouse Recon 1 and Mouse Recon 2

\begin{tabular}{lll}
\hline Property & Recon 1 & Recon 2 \\
\hline Total number of reactions & 4091 & 6172 \\
Total number of metabolites & 2950 & 4380 \\
Number of unique metabolites & 1535 & 2293 \\
Number of metabolites in extracellular space & 469 & 567 \\
Number of metabolites in cytoplasm & 1029 & 1357 \\
Number of metabolites in mitochondrion & 393 & 732 \\
Number of metabolites in nucleus & 95 & 155 \\
Number of metabolites in endoplasmic reticulum & 238 & 505 \\
Number of metabolites in peroxisome & 143 & 422 \\
Number of metabolites in lysosome & 219 & 251 \\
Number of metabolites in Golgi apparatus & 281 & 305 \\
Number of unique genes & 1776 & 1123 \\
Number of subsystems & 102 & $1496(24 \%)^{\mathrm{a}}$ \\
Number of blocked reactions (\% of all reactions) & $789(19 \%)$ & $1181^{\mathrm{a}}$ \\
Number of dead-end metabolites & 510 & $338^{\mathrm{a}}$ \\
\hline Number of metabolic functions (out of 363) & 304 & 214 \\
\hline
\end{tabular}

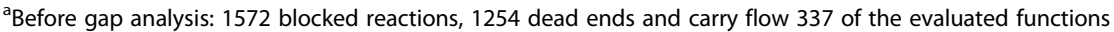

2 (Fig. 1d, e). Out of the total 336 metabolic function tested, OvoFol Recon 1 successfully simulated 246 functions (Additional file 3). A total of 1212 follicular genes that encodes for enzymes were identified in the four transcriptomics datasets (Additional file 9: Table S3) and were also present in Mouse Recon 2, out of these 1212 follicular genes, 1078 were present in OvoFol Recon 1 too. The remaining follicular genes that encode for enzymes did not have any functional metabolic reaction associated with them and were therefore excluded from the reconstruction. This model provides a comprehensive map of the mouse ovarian follicle metabolism that can be mined to identify active metabolic pathways in the female germ cell line and the metabolites that it consumes or secretes.

\section{Network analysis of the mouse ovarian follicle metabolic reconstruction}

Next, we established the major metabolic pathways that were differentially active across multiple follicle stages using a network approach. Superimposing transcriptomic information in enzyme ovarian follicle metabolic network is a powerful approach to identify active metabolic pathways. [21]. We created an enzyme ovarian follicle metabolic network by connecting enzymes that share common metabolites. Highly inter-connected enzymes within a network are called communities. Communities are clusters of members (e.g., enzymes, genes) that have more connections between themselves than with other members in the network. OvolFol Recon 1 was divided into 30 communities (Additional file 6), or clusters of highly interconnected enzymes (Fig. 2), according to Infomap [22, 23], one of the leading community detection methods [24]. Infomap decomposes a network into communities based on what is called information flow through the network. Information flow between enzymes that are closely connected, i.e., they share common metabolites, is greater than between enzymes that do not possess any common metabolites. Hence, communities are formed by enzymes that support high levels of information flow between them.

Subsequently, we overlaid transcriptional data for each ovarian follicle cell type into OvolFol Recon 1. Cell-type specific genes that encode for enzymes were overlapped over OvolFol Recon1 to render oocyte-, somatic-, cumulus- and mural-specific metabolic models. To determine cell-type specific metabolic pathways, we calculated a normalized flow through each community. This normalized flow through each community was quantified using Eq. 1 (Methods), and accounts for differences in community sizes and connection between them; it further accounts for changes in abundance of transcripts encoding enzymes that participate in each community and the number of metabolites that each enzyme catalyzes. Thus, the most transcriptionally-active and highly connected communities in the network will have the largest normalized flow.

Normalized flow revealed metabolic patterns for the multiple cell types and follicle stages during ovarian follicle development (Additional file 9: Figure S3). Notably, the metabolic communities in primordial oocytes differed significantly from that of oocytes at other follicle stages (Additional file 9: Figure S3). Peroxisomal 


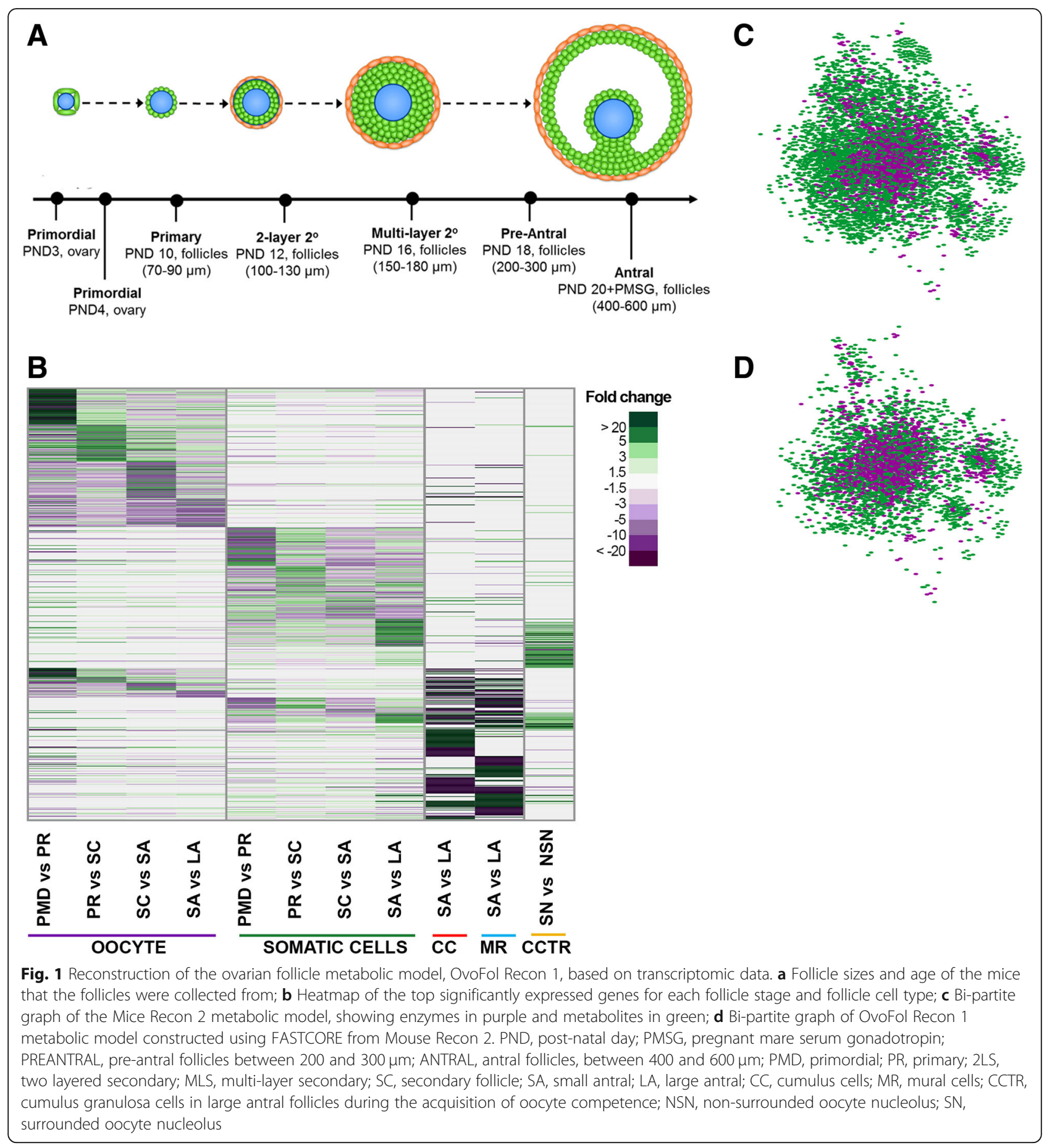

(Community 6) and lysosomal (Community 7) processes were more prominent in the oocyte at the early stages of follicle development, while mitochondrial processes in the oocyte were enhanced over time (Community 9). The enhancement of oocyte mitochondrial processes might be associated with the observations that oxidative phosphorylation is more prominent at the later stages during follicle development once that the antrum is formed [7], as oxygen is more accessible for the oocyte, probably from the follicular fluid $[25,26]$. Compared to the oocyte, the somatic cells (granulosa and theca cells) had greater metabolic activity in the cytosol (Community 1 ) and the endoplasmic reticulum (Communities 4, 12 , and 22), with the latter especially active during the antral stage. When comparing cumulus cells and mural cells during antral formation, cumulus cells showed 


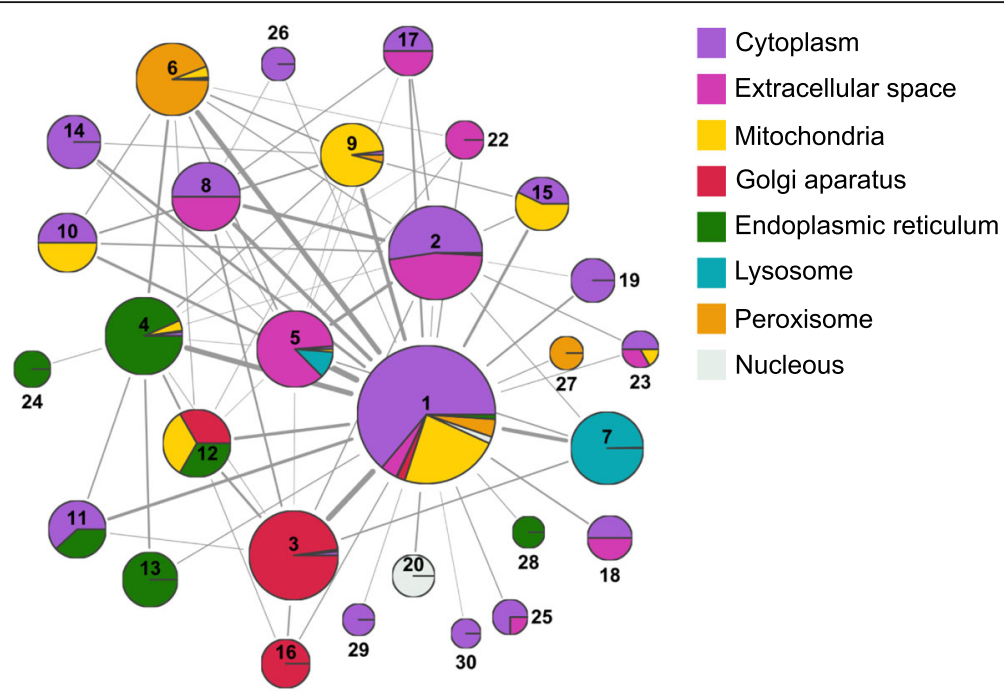

Fig. 2 Ovarian follicle metabolic communities in OvoFol Recon 1 based on metabolite flow between enzymes (from Infomap). The sizes of the communities, defined as clusters of highly connected enzymes based on the flow of information through them, and the width of the edges between communities are proportional to the information that flows through them. Communities are color-coded based on the proportion of enzymes that pertain to a given location. For instance, Community 1 is largely composed of cytoplasmic enzymes (purple), followed by mitochondrial enzymes (yellow)

more metabolic processes activated in the lysosomes (Community 7), whereas mural cells had more activity in the peroxisome (Community 6). Interestingly, during the acquisition of oocyte competence, characterized by the transition from non-surrounded oocyte nucleolus (NSN) to the surrounded oocyte nucleolus (SN), cumulus cells present in large antral follicles during the transition exhibited more prominent peroxisomal processes (Community 6) than lysosomal processes (Community 7). Note that mitochondrial processes were mostly silent in cumulus cells (Community 9) and were only active in the incipient mural cells.

Enriched metabolic pathways during follicle development We next identified metabolic pathways, as defined by the Kyoto Encyclopedia of Genes and Genomes [27], that are over-represented in the cell-type specific metabolic communities. Pathway enrichment was calculated as the normalized flow of all the genes that belong to a specific pathway (e.g., pyruvate metabolism) compared with the background normalized flow of the equal number of randomly selected genes for the entire network (Methods).

Analysis of information flows based on the connectivity of the metabolic network and the transcriptomic data allowed identification of the most enriched metabolic pathways in the cell-specific oocyte and somatic cell metabolic models during follicle development (Fig. 3). The enrichment analysis identified well-known metabolic pathways that occur during follicle development, such as pyruvate metabolism in the oocyte [26], the production of estrogen in granulosa cells in antral follicles
[26], and the production of bile acids [28]. Moreover, we were also able to pinpoint new or less studied pathways, such as folate acid metabolism in somatic cells, starch and sucrose metabolism in the oocyte, and limonene and pinene degradation in the mural cells.

Most of the enriched metabolic pathways belonged to Communities 1 and 2, according to the most active and differentiated pathways among diverse cell types (Additional file 7). At the primordial stage, the enriched metabolic pathways in the oocyte were arginine and proline metabolism and oxidative phosphorylation, whereas folate metabolism and fatty acid oxidation were among the most active metabolic pathways in primordial somatic cells -note that in this case somatic cells includes squamous granulosa cells and stroma cells that surround the oocyte. Fatty acid oxidation was active in the primordial follicle, both in the oocyte and in the somatic cells, providing a source of energy for the early follicle. This observation is consistent with previous studies that have suggested that glycogen is one of the sources of energy in primordial germ cells [26]. In contrast, the top significant metabolic pathways in oocytes in primary, secondary, and antral follicles were coenzyme catabolism and fatty acid oxidation in the peroxisome. Somatic cells of primary, secondary, and antral follicles had highly active metabolism of folic acid and nucleotides, extracellular transport (Community 2), and heme production.

During the formation of the antrum and the differentiation of granulosa cells into cumulus and mural cells, the ranking of metabolic pathways in terms of their Z-scores changed with respect to the other cell types. Inosinic acid and carnitine shuttling were among the top 


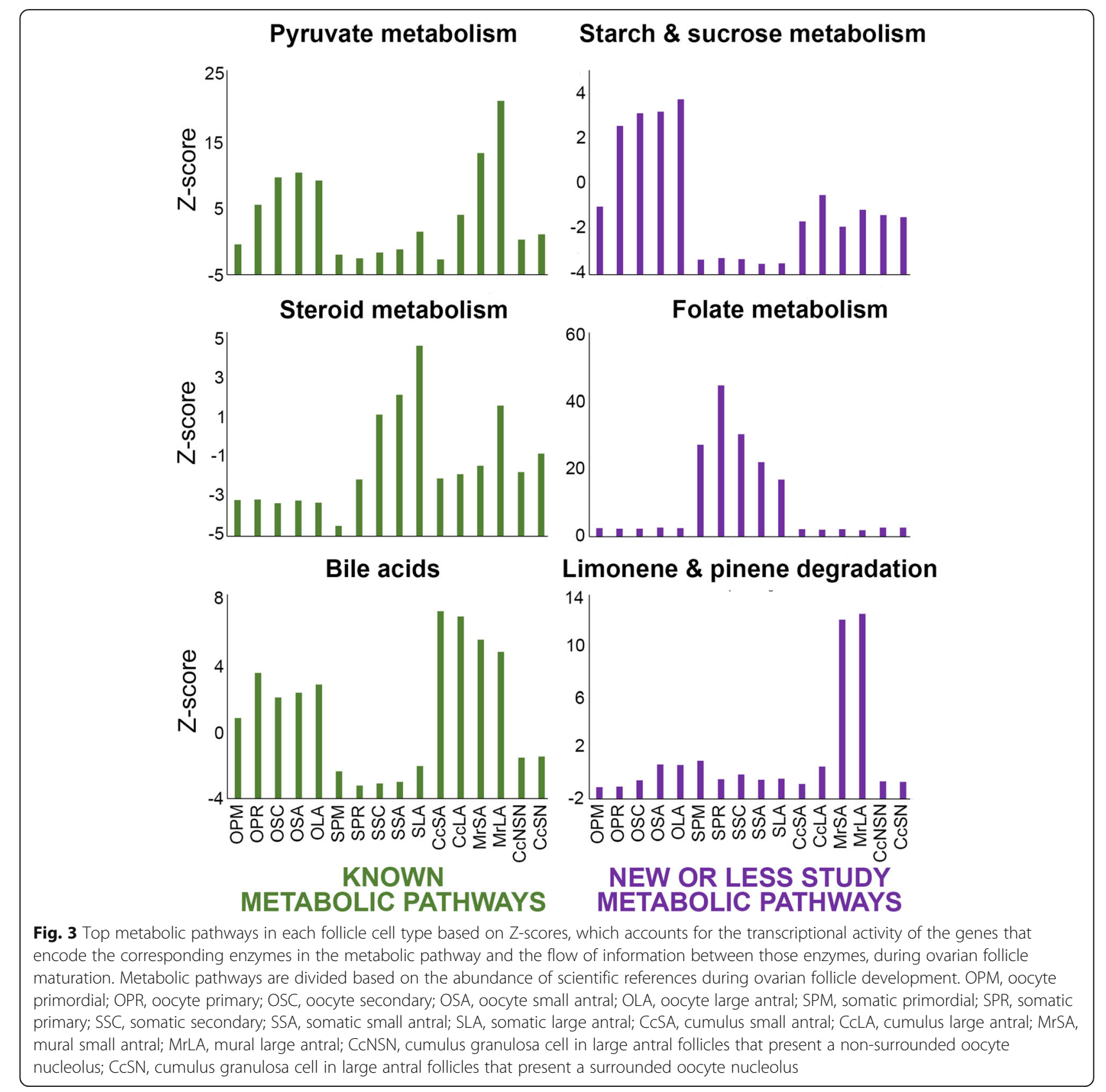

metabolic pathways in cumulus cells; in contrast, mural cells showed highly active pyruvate metabolism, limonene and pinene degradation, o-glycan synthesis, and transport to the lysosome. Finally, during the acquisition of oocyte competence, the most active pathways in cumulus cells were fatty acid oxidation and cholesterol and propionate metabolism.

Key metabolites during mouse ovarian follicle maturation We next identified the most significant metabolites using a similar approach to the identification of over-represented pathways. Key metabolites were uncovered by measuring the total information flow through the enzymes that catalyze the reactions in which the metabolite participates (Methods). We identified the top 10 metabolites for each follicle stage and cell type (Fig. 4a). Top metabolites for oocytes in primordial follicles did not align with those in oocytes in other follicle stages. For instance, nitric oxide (NO) in the cytosol and the intracellular cytosolic and extracellular calcium ion $\left(\mathrm{Ca}^{2+}\right)$ were the most important metabolites in primordial oocytes, while 6-phosphoD-glucono-1,5-lactone and 2,3-bisphosphonato-D-glycerate, which are involved in the pentose phosphate 


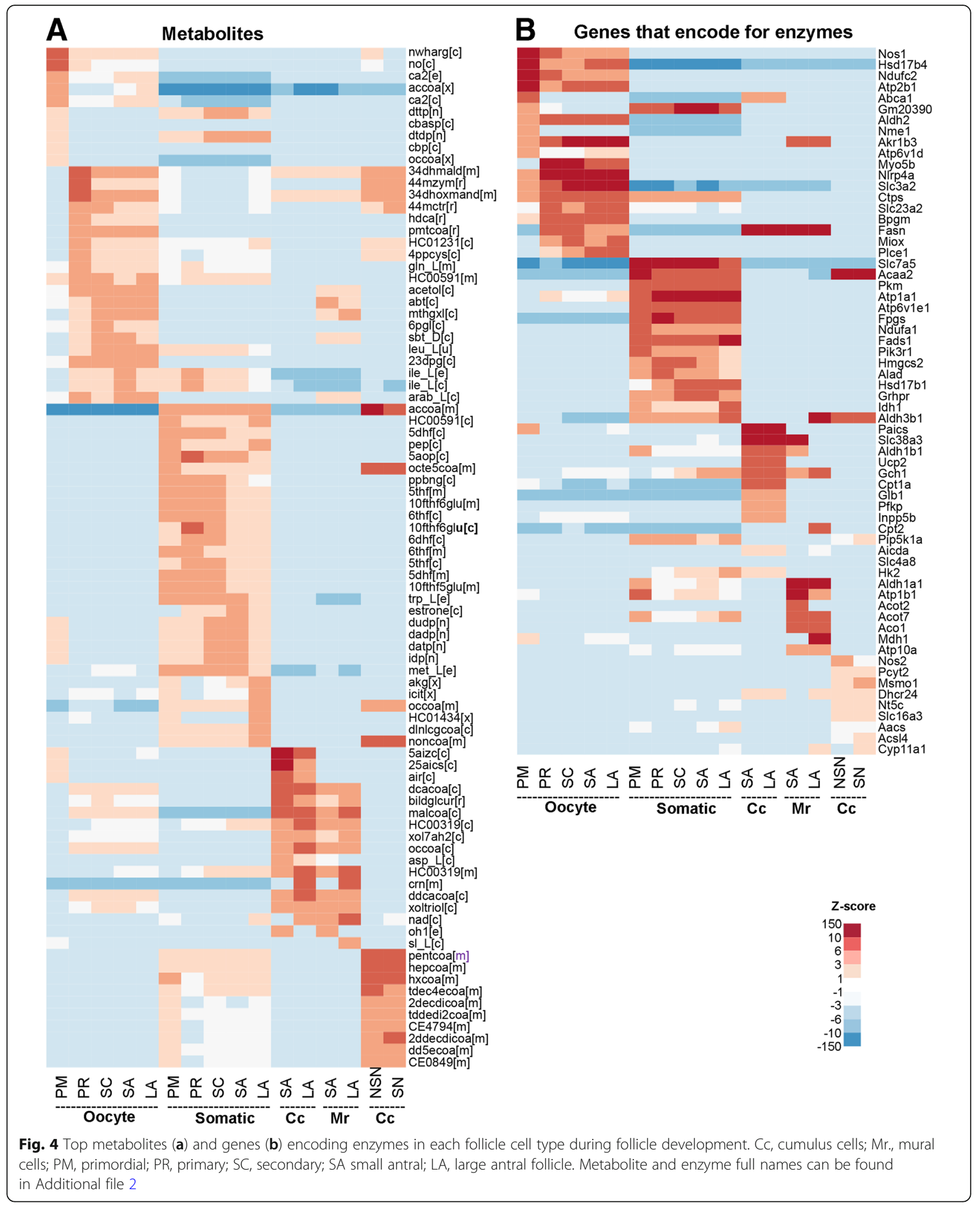

pathway and oxygen release from red blood cells, respectively, were more prominent in oocytes at later follicle stages (Fig. 4a).
Interestingly, significant metabolites in the somatic cells were similar at all follicles stages and were primarily folic acid derivatives and L-methionine, which are 


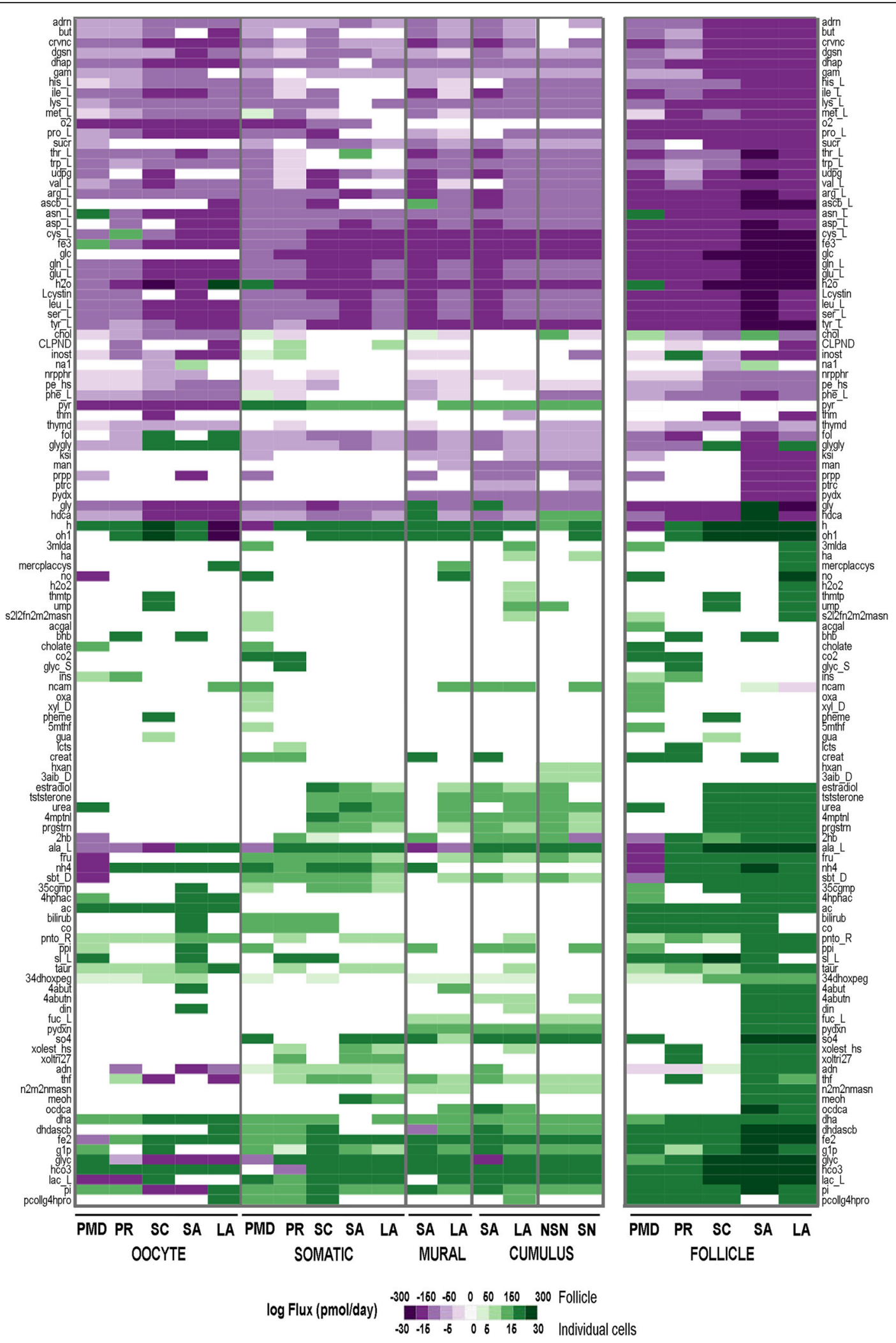

Fig. 5 Predicted exo- and endo-metabolism in each follicle cell type during follicle maturation. PMD, primordial follicle; PR, primary; SC, secondary; SA, small antral follicle; LA, large antral follicle; NSN, non-surrounded oocyte nucleolus; SN, surrounded oocyte nucleolus. Extracellular reactions and metabolite full names can be found in Additional file 2 
involved in DNA methylation. L-tryptophan had a high enrichment Z-score in somatic cells as well. This amino acid is the precursor of serotine, which has known effects in follicle maturation [29]. Cytosolic estrone did not become a significant metabolite in the somatic cells until the secondary stage and onwards, as expected. Cumulus granulosa cells during the small to large antral transition showed a significant activation of metabolites related to purine metabolism (e.g., 5aizc, 25aics, air). Lipids are known to play an important role during the acquisition of oocyte competence [30], and were indeed overrepresented in the cumulus cells during the NSN to $\mathrm{SN}$ transition in the oocyte to acquire its competence (e.g., pentanoyl-coa, heptanoyl-coa, hexanoyl-coa).

\section{Key genes that encode for enzymes during follicle maturation}

Similarly, we identified the top 10 genes encoding enzymes in each cell type and follicle stage based on their Z-scores (Fig. 4b). The most significantly expressed genes encoding enzymes in the oocytes of primordial follicles differed from those expressed in oocytes at other follicle stages. Nos1 (nitric oxide synthase) and Hsd17b4 (hydroxysteroid 17-beta dehydrogenase 4), which is an enzyme part of the peroxisomal beta-oxidation pathway for fatty acids, were the two top enzymes in primordial oocytes; whereas Myo5b (Myosin Vb), an effector for RAB11A required for recycling of transferrin in nonpolarized cells [31], Akr1b3 (aldo-keto reductase family 1, member B3), which participates in pyruvate metabolism, and Scl3a2, a glutamine transporter, were among the most enriched genes encoding enzymes in oocytes of all other follicle stages (Fig. 4b).

In the somatic cells, the top most significant genes encoding enzymes based were Slc7a5, Atp1a1 (ATPase $\mathrm{Na}+/ \mathrm{K}+$ transporting subunit alpha 1), Fpgs (folylpolyglutamate synthase), and Fdas1 (fatty acid desaturase 1). Slc7a5 encodes an amino acid transporter involved in high-affinity transport of large neutral amino acids such as phenylalanine, tyrosine, leucine, arginine, and tryptophan, while Fpgs encodes an enzyme that establishes and maintains both cytosolic and mitochondrial folylpolyglutamate concentrations and, therefore, is essential for folate homeostasis and the survival of proliferating cells. The enzyme encoding by Fpgs catalyzes the conversion of folates to polyglutamate derivatives allowing to maintain the concentrations of folate components in the cell. Fpgs also facilitates the intracellular retention of these cofactors, which are important substrates for most of the folate-dependent enzymes that are involved in one-carbon transfer reactions in purine, pyrimidine, and amino acid synthesis. Fdas1 Isoform 1, which has its highest Z-score values in the large antral follicles, encodes a component of a lipid metabolic pathway that catalyzes the biosynthesis of highly unsaturated fatty acids and generates arachidonic acid. Arachidonic acid increases the concentration of estrogen and progesterone in granulosa and theca cells [32]. Hsd17b1 (hydroxysteroid 17-beta dehydrogenase 1) encodes an enzyme involved in the metabolism of estrogens, and reduces both estrogens and androgens (Fig. 4b). Highly ranked genes in cumulus cells were Paics, which is involved in purine biosynthesis, and Aldh1a1 (aldehyde dehydrogenase 1 family member A1) in mural cells, a gene that encodes an enzyme that produces retinoic acid, an important vitamin component in ovarian follicle development [33].

\section{Exo- and endo-metabolites during in vivo follicle maturation}

Finally, we determined the most likely secreted and consumed metabolites by each cell type at each follicle stage (Fig. 5). We simulated each stage- and cell-type specific metabolic models, using experimentally measured metabolites that are consumed or produced during follicle development (e.g., glucose, oxygen, Additional file 9: Table S4) to constrain the metabolic models. The plasma composition of these metabolites was employed for these calculations (Additional file 9: Table S5). Our computational results were consistent with several reports on multiple metabolites: i) the consumption of nitric oxide by the oocyte, which prevent apoptosis [34]; ii) consumption of fructose, sorbitol, and L-lactose [26] by the oocyte, which somatic cells produce; iii) L-alanine uptake by oocytes and secretion of L-alanine at later stages [35]; iv) hypoxanthine production in cumulus cells during the time of the oocyte is acquiring competence to inhibit oocyte maturation [36, 37], as well as the production of L-fucose [38]; and iii) collagen production only by the somatic cells that requires ascorbic acid, which has been recently demonstrated by our group [39]. Ascorbic acid allows the survival of smaller follicles by supporting the production of more extracellular matrix (ECM) components [39]. Supplementation of the $\alpha$-MEM media that is currently used in in vitro follicle culture has shed some light into the long-standing challenge of growing primary follicles in 3-D alginate gels [39].

Our analysis also discovered novel metabolic processes during ovarian follicle maturation. For example, our model suggests that the oocyte produces metabolites acetate and inosine. Acetate is the source of cholesterol for cumulus cells [40], and based on our computational results, the origin of that acetate is the oocyte itself. Inosine maintains the meiotic arrest of oocytes [36], but it is also produced by the oocytes themselves, and not by the cumulus cells, as it happens with hypoxanthine. Somatic cells, from primordial follicles all the way to 
antral follicles, consumed folic acid. Additionally, the oocyte produced folic acid, specifically at the later stages of ovarian follicle development. Another similar component, vitamin B6 (pyridoxine) was produced by the cumulus and mural cells. At the follicle level, the model predicted that most of the amino acids were consumed except for the non-essential amino acid taurine, and sucrose.

\section{Discussion}

In this study, we created and analyzed the first metabolic model of the mouse ovarian follicle and its cellular compartments - the oocyte and somatic cells (i.e., granulosa and theca cells) - through the stages of follicle development. We used a systems biology approach to decipher the key exo- and endo-metabolic processes present during mouse ovarian folliculogenesis in vivo. For this purpose, the latest mouse metabolic reconstruction, Mouse Recon 1, was updated based on the recently developed human reconstruction, Human Recon 2. This new model was able to successfully simulate an additional $10 \%$ of the metabolic functions compared to the Mouse Recon 1 model. Mouse Recon 2 was then employed to generate the first mouse ovarian follicle metabolic reconstruction, OvoFol Recon 1. Mouse Recon 2 could be applied in future studies to predict mouse phenotypes using IMPC [41] (http://www.mousephenotype.org/). Similarly, OvolFol Recon 1 could seed light into human diseases associated with ovarian follicle development (e.g., https://rarediseases.info.nih. gov/diseases/diseases-by-category/10/female-reproductive-diseases).

Our new method based on the combination of network approaches and transcriptional activity identified the most relevant metabolic pathways, metabolites and metabolic genes. We were able to identify cell-specific metabolic pathways that occur during follicle development (e.g., pyruvate metabolism in the oocyte [26], the production of estrogen in granulosa cells in antral follicles [26] and the production of bile acids [28]). Our approach also identified new or less studied pathways, such as folate acid metabolism in somatic cells, starch and sucrose metabolism in the oocyte, and limonene and pinene degradation in the mural cells.

Our new methods allowed the exploration of the variation in metabolites and enzymes and may support future studies on the communication between the multiple cellular compartments within the follicle and the metabolic changes within other multi-cellular systems. For instance, we were able to reveal two interesting examples in ovarian follicle metabolism: folic acid and taurine. Folate metabolism has been extensively studied due to its implications on fetal outcomes, as it alters the DNA methylation profiles of the oocyte together with methionine [42], but the origins and effects of folate during follicle formation are relatively unknown. In fact, our model suggests the follicle consumes folic acid. Taurine has been measured before in the mouse follicular fluid (Additional file 9: Table S5), and it was speculated that the large concentration of taurine in the follicular fluid was due to its accumulation, as it was not consumed by the follicle [6]. Based on our model, taurine is produced by the oocyte and the somatic cells beginning from the primary stage, and even in the primordial somatic cells. Taurine has several physiological actions [43] - it is a potent anti-oxidant [44] and intervenes in calcium transport [45]. At this point, the effects of taurine in ovarian follicle maturation are not clear; however, it is tempting to hypothesize that taurine is produced by the oocyte and somatic cells to protect the oocyte against radical oxidant species and improve calcium transport required for downstream signaling of follicle-stimulating hormone (FSH).

Identification of the metabolites that are secreted or consumed by the ovarian follicle during its development has two-fold significance: a) secreted metabolites may be employed as biomarkers for the stage of follicle development, which is necessary to monitor the growth in vitro of follicles from large mammalian species whose stage cannot be monitored under the microscope. Several metabolites may be useful for determining follicular stage. The transition from primordial follicles to primary follicles could be detected by the decrease of production of nitric oxide, oxalic acid, D-xylose, cholate, or 5-methyltetrahydrofolate. Similarly, the primary to secondary transition could be monitored based on the decrease in production of inosine or the production of (R)-3-hydroxybutyrate; b) consumed metabolites can guide the development of novel media components that will support follicle growth, particularly in early-stage follicles, and in vitro maturation (IVM). For instance, based on the model predictions, supplementation of the $\alpha$-MEM media that is currently used in in vitro follicle culture with sorbitol and 2-hydroxybutyrate or further increase the concentration of folic acid in the media, as the three metabolites are consumed by the follicles.

The metabolome of the oocyte differed greatly from that of the somatic cells at every stage of follicle development. This result is consistent with current thinking regarding the geography of the ovary, where primordial follicles, which are located in the ovarian cortex, and secondary and antral follicles, located in the medulla [46], are exposed to different metabolites. The limited success in growing primordial follicles in vitro without using two-step cultures [47] may be related to the distinct metabolic differences between early and later-stage follicles. In fact, current in vitro follicle culture approaches were developed based on later-stage follicles 
where a relatively large body of knowledge of their biology is available [26]. These media are being ineffectively applied to designed primordial follicle growth media and conditions. Tuning the media composition as the ovarian follicle grows and the oocyte matures in culture may ultimately enhance oocyte quality.

The aim of our study was to develop novel data-driven hypothesis that could serve as new areas of research to understand the complex and dynamic intra- and intercellular communication between the different ovarian follicular cell types. Our models can be improved by using experimental isolated cell types from all the stages during follicle development. Currently, there is no such a set in mice, especially for granulosa cells, most likely due to the technical difficulties. Similarly, it should be noted that the results from our model have been obtained using transcriptional data from pre-pubertal mice and those differ from pubertal mice, as the dynamics of ovarian follicle development is faster in younger mice and slows with mouse age [48]. Finally, predictions obtained from transcriptomic data alone does not guarantee the activity of a given enzyme. Herein, mRNA levels were employed as a proxy for enzymatic activity, while metabolomics and proteomics would be ideal [12, 13]. Established non-target metabolomic techniques typically require millions of cells [49], and even emerging techniques require on the order of thousands of cells [50], which is prohibited for oocyte isolation. Proteomics approaches that account for post-translational modifications would be desirable, however, non-proteomics data is only available at later ovarian folliculogenesis stages for entire follicles grown in vitro [51]. In fact, the combination of proteomics with the community network approach reported herein would have the potential to reveal the most important systems and key metabolites and enzymes, as it has been done before in human tissues [52].

\section{Conclusion}

In conclusion, we successfully applied a systems biology approach to characterize the most important metabolic pathways in the oocyte and somatic cells during various stages of ovarian follicle development. The understanding of the follicle metabolome has been limited by decades of research using bottom-up approaches, which has provided only snapshots of the complex metabolic landscape of the growing follicle. Collectively, metabolic systems approaches were able to model the follicle metabolome, providing a rich set of data that can be applied to generate new hypothesis to test experimentally. Our study can improve in vitro follicle growth and oocyte maturation approaches and support the growth of primordial follicles in vitro.

\section{Methods}

\section{Updating the mouse general metabolic model}

We developed Mouse Recon 2, a general metabolic model based on the latest general human metabolic reconstruction, Human Recon 2.03 [53], employing a similar methodology to that applied for the first mouse metabolic reconstruction, Mouse Recon 1 [54]. The reconstruction was performed through a series of iterative steps aiming to reduce ambiguities when merging the two reconstructions, Mouse Recon 1 and Human Recon 2.03 (Additional file 8: Note S1) and was followed by a gap analysis evaluation using fastGapFill [55] (Additional file 8: Note S2). Mouse Recon 2 was examined against a total of 363 metabolic functions, such as production of biomass, production of pyruvate and lactate under anaerobic conditions from glucose, pyruvate consumption, hormone production (estrogen, androgen, testosterone, and progesterone), and fructose and sorbitol production (Additional file 3). Flux variance analysis was subsequently performed to determine the dead-end metabolites and blocked reactions (i.e., reactions that either their reactants are not produced by any other reactions or obtained from the cell media, or their metabolites are not consumed neither exported outside the cell). The resulting MATLAB Mice Recon 2 model is provided in Additional file 1, and the model reactions, metabolites, and genes are summarized in Additional file 2.

\section{Follicle collection and isolation}

We followed procedures for ovary and follicle isolation as previously established [56] with slight modifications. CD-1 mice were obtained commercially from Harlan Laboratories, USA. CD-1 mice were housed in a temperature- and light-controlled environment (14 h light, $12 \mathrm{~h}$ dark) and provided with food and water ad libitum. Animals were fed Teklad Global irradiated 2919 low-phytoestrogen chow. At the time of delivery, 8 female pups were housed with each dam to minimize differences in pup development caused by nutrient availability. Animals were treated in accordance with the NIH Guide for the Care and Use of Laboratory Animals and the established IACUC protocol at Northwestern University. Donor mice were euthanized by $\mathrm{CO} 2$ inhalation followed by cervical dislocation. Entire ovaries were collected at post-natal day 3 and day 4 to collect primordial follicles. Primary follicles $(70-90 \mu \mathrm{m}$ in diameter), two-layered secondary follicles $(100-130 \mu \mathrm{m})$, multi-layer secondary follicles $(150-180 \mu \mathrm{m})$, and pre-antral follicles $(200-300 \mu \mathrm{m})$ were mechanically isolated from post-natal day 10, 12, 16, and 18 ovaries, respectively. Antral follicles $(400-600 \mu \mathrm{m})$ were mechanically isolated from pregnant mare serum gonadotropin (PMSG)-treated mice ovaries at post-natal day 20. 
Follicles were then aspirated and combined per ovarian follicle maturation stage (e.g., primary, two-layered secondary). Three different samples were collected from each pooled follicular stage for transcriptomic analysis. RNA was purified and hybridized in MouseRef-8 v2.0 Expression BeadChip Kit (Illumina, San Diego, CA), as previously described [57].

\section{Follicle transcriptome data}

Microarray data were downloaded from Gene Expression Omnibus (GEO) using the GEOquery [58] and Array Express packages [59] from Bioconductor (http:// www.bioconductor.org) for: a) mouse oocytes from primordial to large antral follicles [18] (E-GEOD-3351); b) mouse cumulus and mural cells collected during antrum formation (secondary to antral transition) [19] (GSE55845), and c) mouse cumulus cells collected at the time of oocyte competence acquisition (large antral follicles) [20] (E-GEOD-36617). The three published microarray datasets and our microarray data from the isolated mouse primordial to large antral follicles, as described above, were normalized and transformed and non-detected probes were removed as indicated in Additional file 9: Table S3 [60]. Significant genes were identified using limma [61] and were corrected for multiple comparisons using the false discovery rate (fdr) method [62]. Our microarray data are published as GSE97902.

\section{mRNA segregation process}

Genes present in both oocyte and the follicle transcriptome were removed from the follicle transcriptome before statistical analysis if they met the two following conditions: a) they were significant in the oocyte microarray ( $\mathrm{FC} \geq 2.5$ and fdr-corrected $p$-value $\leq 0.01$ ); b) and their fold change in the follicle microarray was below 1.05 from for the primary to two-layered secondary transition- the oocyte transcripts are diluted due to the granulosa cell proliferation. The genes that satisfied these conditions were considered to be only expressed in the oocyte during ovarian follicle development. Genes in the follicle microarrays that were significant in the oocyte and in the follicle microarray but did not follow the patterns described in conditions $b$ and $c$ were classified as present in both cell types, i.e., oocyte and somatic cells (Additional file 8). The rest were classified as genes that are only transcribed in the somatic cells and their experimentally determined transcription abundance was employed in all the later calculations.

Genes from each microarray were classified as oocyte only, somatic only (granulosa and theca cells), cumulus cell only, cumulus cell during acquisition of oocyte competence only, or mural cell only if they were present only in their corresponding arrays and were not significant in any other microarray for the given significance cut-offs
(Additional file 9: Table S3). Those genes that did not satisfy the above conditions were considered to be present in multiple cell types (e.g., if a gene A was significant in the oocyte and cumulus cells).

\section{Follicle metabolic reconstruction and analysis}

To generate the mouse ovarian follicle metabolic reconstruction, which we named OvoFol Recon 1, we used ovarian follicle transcriptomic data (Additional file 9: Table S3) and the FASTCORE algorithm [17]. First, the relevant genes included in Mouse Recon 2 were identified by removing all the blocked reactions using the fastcc function from FASTCORE. Then, a consistent cell-specific mouse ovarian follicle reconstruction was established with the fastcore function. OvoFol Recon 1 was validated against 363 metabolic functions using plasma composition (Additional file 9: Table S5).

An enzyme-metabolite bi-partite graph and an enzyme network graph were constructed based on the resulting OvoFol Recon 1. The enzyme-metabolite bi-partite graph included connections between enzymes and the corresponding metabolites they catalyze. The enzyme network graph contained only edges between the enzymes that catalyze the same metabolite. The number of communities, defined as clusters or partitions of highly interconnected enzymes, in the OvoFol Recon 1 enzyme network graph was established with Infomap [22, 24] using 1000 iterations. Infomap decomposes a network into communities based on a description of information flows in the network. Information flow between enzymes that are closely related, i.e., based on common metabolites, is greater than between enzymes that do not possess any common metabolites. Hence, communities are formed by enzymes that support high levels of information flow between them. The normalized flow through each community was calculated using Eq. 1, which accounts for differences in community sizes as well as the dynamic changes in mRNA abundance of the genes encoding enzymes that participate in each community. The normalized flow through the community was calculated as follows:

$$
f_{N_{i}}=\frac{\sum_{k=1}^{k=n_{i}} w_{k} I_{k} f_{k}}{\sqrt{\sum_{k=1}^{k=n_{i}} w_{k}}}
$$

where $f_{N i}$ is the normalized intensity flow of the community (or pathway, or metabolite) $i, f_{k}$ is the flow calculated with Infomap for element $k$ in the community $i, w_{k}$ is the number of metabolites that are catalyzed by the enzyme $k$ according to the enzyme-metabolite bi-partite graph, $I_{k}$ is 1 if no microarray data was employed (Fig. 2), 
or the intensity value for the given gene in the transcriptomic data (Additional file 9: Figure S3). This normalized flow accounts for differences in community sizes as well as the dynamic changes in mRNA abundance of the genes encoding enzymes that participate in each community. By weighting the number of metabolites that each enzyme catalyzes, we accounted for the impact that each enzyme has at the metabolic level.

\section{Metabolic hot spots: communities, pathways, genes and metabolites}

The most significant communities or "hotspots" in OvoFol Recon 1 were uncovered by measuring the total flow per community, normalized by the community size (Eq. 1). Z-scores for the metabolic pathways, metabolites, and genes were obtained by calculating the corresponding mean, $\mu$, and standard deviation, $\sigma$, of 1000 randomly samples of the same size (Eq. 2). The values of the samples corresponded to randomly shifting the normalized intensity flows among all the elements without replacement.

$$
Z-\text { score }_{i}=\frac{\mathrm{f}_{p}-\mu}{\sigma}
$$

Pathway enrichment within a community, $f_{p}$, was calculated as the normalized intensity flow of all the genes or nodes in a given community that belong to a specific pathway within OvoFol Recon 1 (e.g., pyruvate metabolism). $f_{p}$ was compared with the background normalized intensity flow of the equal number of randomly selected genes for the entire network using the enzyme-metabolite bi-partite graph. Enzyme Z-scores were established using Eq. 1 , and $f_{p}$ was determined based on the flow of the enzyme accounting for all the metabolites that are catalyzed by the given enzyme in the entire network, $w_{k}$. Similarly, Z-scores of the metabolites were established using Eq. 1 and $f_{p}$ was determined based on the flow of the enzymes that catalyze the reactions in which the metabolite participates, setting $w_{k}$ to 1 .

\section{Number of granulosa, theca, and cumulus cells during ovarian follicle development in vivo}

Deparaffinized histological sections of the ovaries employed for the in vivo transcriptomic studies were used to determine the number of different cell types over time in each follicle stage (Additional file 9: Figure S2). The total number of granulosa cells, $n_{G}$, was calculated as follows [63] (Eqs. 3 and 4):

$$
n_{G}=\frac{4}{3} \pi \rho_{G}\left(r_{F w o T}^{3}-r_{O}^{3}-r_{A}^{3}\right)
$$

$$
r_{A}=\sqrt{\frac{A_{A}}{\pi}}
$$

where $\rho_{G}$ is the granulosa cell density; $r_{F w o F}$ is the radius radio of the follicle excluding the theca layer if present; $r_{O}$ is the oocyte radius; $r_{A}$ is the antral radius that was estimated assuming that the observed antral area; and $A_{A}$, when present, was a sphere. The granulosa cell density was based on the observed granulosa volume in the image, taking into account that the slides were $5 \mu \mathrm{m}$ thick, $f$, by the total number of manually counted granulosa cells in the slide (Eq. 5).

$$
\rho_{G}=\frac{f \pi\left(r_{F w o T}^{2}-r_{O}^{2}-A_{A}\right)}{n_{\text {Ginslide }}}
$$

The total number of theca and cumulus cells were estimated using the same approach. The cell type ratios were obtained from slides in which the nucleus of the oocyte could be observed. The average of the maximal and minimal axis of the follicle was employed as an estimation of the cellular ratios. All images were processed with ImageJ (Rasband, W.S., ImageJ, U. S. National Institutes of Health, Bethesda, Maryland, USA, http://imagej. nih.gov/ij/, 1997-2014).

\section{Cell-specific models and follicle exo- and endo- metabolism analysis}

Metabolic networks within and between the oocyte and somatic cells during each stage of ovarian follicle development (primordial, primary, secondary, antral, and non-surrounding and surrounding nucleolus) were reconstructed using the FASTCORE algorithm [17] in the same manner as OvoFol Recon 1. For each of these follicle stage-specific reconstructions, the secreted metabolites were obtained (Additional file 9: Supplementary Note 3) and constrained with experimental parameters when they were available (Additional file 9: Table S4). Previously experimentally measured oxygen, glucose, and lactose consumption or production, hormone production, and hyaluronic acid production were collected from various sources in the literature (Additional file 9: Table S4). The plasma composition of metabolites was employed for these calculations (Additional file 9: Table S5). Total metabolite flow uptake was determined iteratively, so that the final estimated pyruvate flux coincided with the experimentally measured pyruvate production by the somatic cells. Metabolites produced or consumed by the somatic cells were added to or subtracted from the initial flux to the oocyte. The number of somatic cells (granulosa and theca cells) at each follicular stage was accounted for, and the updated flux was utilized to determine the metabolic behavior of the oocyte. For the somatic cells, flux balance analysis (FBA) was run by 
setting the objective function to maximize the pyruvate and biomass production subject to the experimental values (e.g., glucose intake, production of lactic acid, estrogen, hyaluronic acid). Except for primordial somatic cells, oxygen was restricted to be only consumed for somatic cells. Production of nitric oxide, carbon monoxide, L-alanine were included at all the somatic cell stages; sorbitol and fructose were added to the secondary to antral follicle objective functions, and hormone production (progesterone, testosterone and estrogen) and hyaluronic acid were included in the antral follicle objective function only. For the oocyte, the optimization function was composed of the biomass production and pyruvate consumption, subject to the oxygen consumption measured experimentally and consumption/production of lactic acid and consumption of sorbitol, fructose and ascorbic acid.

\section{Additional files}

Additional file 1: Mice Recon 2, Matlab file

Additional file 2: Metabolites, reactions and enzymes present in Mice Recon 2. (XLSX $452 \mathrm{~kb})$

Additional file 3: Metabolic model functionalities. (XLSX 66 kb)

Additional file 4: OvoFol Recon 1, Matlab file

Additional file 5: Metabolites, reactions and enzymes present in OvoFol Recon 1. (XLSX 307 kb)

Additional file 6: Entrez ID, gene symbol, the community that the gene belongs to according to Infomap and the flow of information that is transferred through the enzymatic node. (XLSX $110 \mathrm{~kb}$ )

Additional file 7: Z-scores for each metabolic pathway using Infomap information for Mice Recon 2 and OvoFol 1. (XLSX 92 kb)

Additional file 8: Mean fold change and fdr-corrected $p$-values for each follicle cell type during ovarian follicle development. (XLSX 6750 kb)

Additional file 9: Supplementary Figures. (DOCX 13578 kb)

\section{Abbreviations}

25aics: (S)-2-[5-Amino-1-(5-phospho-D-ribosyl)imidazole-4-

carboxamido]succinate; 2LS: Two layered secondary; 5aizc: 5-amino-1-(5phospho-D-ribosyl)imidazole-4-carboxylate; air: 5-amino-1-(5-phospho-Dribosyl)imidazole; Akr1b3: Aldo-keto reductase family 1, member B3; Aldh1a1: Aldehyde dehydrogenase 1 family member A1; ANTRAL: Antral follicles; Atp1a1: ATPase Na+/K+, transporting subunit alpha 1; Bmp15: Bone morphogenetic protein 15; $\mathrm{Ca}_{2}^{+}$: Calcium ion; CC: Cumulus cells; CCLA: Cumulus large antral; CCNSN: Cumulus granulosa cell in large antral follicles that present a non-surrounded oocyte nucleolus; CcSA: Cumulus small antral; CcSN: Cumulus granulosa cell in large antral follicles that present a surrounded oocyte nucleolus; CCTR: Cumulus granulosa cells between the non-surrounded to surrounded nucleolus; COBRA: Constraints-based reconstruction and analysis; ECM: Extracellular matrix; FBA: Flux-balance analysis; FC: Fold change; Fdas1: Fatty acid desaturase 1; fdr: False discovery rate; Fpgs: Folylpolyglutamate synthase; GEO: Gene Expression Omnibus; Hsd17b1: Hydroxysteroid 17-beta dehydrogenase 1; Hsd17b4: Hydroxysteroid 17-beta dehydrogenase 4; IACUC: Institutional Animal Care and Use Committee; IMP: International mouse phenotyping consortium; IVF: In vitro fertilization; IVM: In vitro maturation; LA: Large antral; LP: Linear programming; MLS: Multi-layer secondary; MR: Mural cells; MrLA: Mural large antral; MrSA: Mural small antral; Myo5b: Myosin Vb; NO: Nitric oxide; Nos1: Nitric oxide synthase; NSN: Non-surrounded oocyte nucleolus; OLA: Oocyte large antral; Ooep: Oocyte expressed protein; OPM: Oocyte primordial; OPR: Oocyte primary; OSA: Oocyte small antral; OSC: Oocyte secondary; Paics: Phosphoribosylaminoimidazole carboxylase and phosphoribosylaminoimidazolesuccinocarbox-amide synthase;

PCOS: Polycystic ovarian syndrome; PINs: Protein-protein interaction networks; PMD: Primordial; PMSG: Pregnant mare serum gonadotropin; PND: Post-natal day; PR: Primary; PREANTRAL: Pre-antral follicles; RAB11A: Rasrelated protein Rab-11A; SA: Small antral; SC: Secondary follicle; Scl3a2: Solute carrier family 3 member 2; SLA: Somatic large antral; SIc7a5: Solute carrier family 7 member 5; SN: Surrounded oocyte nucleolus; SPM: Somatic primordial; SPR: Somatic primary; SSA: Somatic small antral; SSC: Somatic secondary; Zp1: Zona pellucida glycoprotein 1; a-MEM: Alpha modified minimum essential medium

\section{Acknowledgments}

We would like to thank Dr. Stacey Tobin for reviewing the manuscript.

\section{Funding}

This work has been mainly supported by NIH/NICHD 2 U54 HD041857-07 for study design and collection, analysis and interpretation of the data. BPB was supported NIH/NIGMS 2 T32 GM008449-16. IT was supported by the Luxembourg National Research Fund (FNR) ATTRACT program grant (FNR/ A12/01). None of the funders, NIH/NICHD, NIH/NIGMS nor FNR played any role in the design of the study and collection, analysis, and interpretation of data nor in writing the manuscript.

\section{Availability of data and materials}

Our in vivo follicle microarray data are published in the Gene Expression Omnibus database (https://www.ncbi.nlm.nih.gov/geo/) as GSE97902. Mouse Recon 2 and OvolFol Recon 1 MATLAB models are available upon request to the authors (Beatriz Penalver Bernabe, bpenalver@gmail.com).

\section{Authors' contributions}

BPB, TKW, LJB and LDS conceived the study; EG and AS collected, isolated and processes the ovarian follicles; BPB and IT developed Mouse Recon 2; BPB performed all the computational studies; BPB, TKW, LJB and LDS interpreted the results and wrote the paper. All authors reviewed the manuscript. All authors read and approved the final manuscript.

\section{Ethics approval and consent to participate}

Animals were treated in accordance with the NIH Guide for the Care and Use of Laboratory Animals and it was approved by the Institutional Animal Care and Use Committee Office at Northwestern University. We employed the established Institutional Animal Care and Use Committee protocol at Northwestern University.

\section{Consent for publication}

Not applicable.

\section{Competing interests}

The authors declare that they have no competing interests.

\section{Publisher's Note}

Springer Nature remains neutral with regard to jurisdictional claims in published maps and institutional affiliations.

\section{Author details}

'Department of Pediatrics, University of California San Diego, La Jolla, CA 92093, USA. ${ }^{2}$ Luxembourg Center for Systems Biology, University of Luxembourg, Esch-sur-Alzette, Luxembourg L-4365, Luxembourg. ${ }^{3}$ Department of Obstetrics and Gynecology, Northwestern University Feinberg School of Medicine, Chicago, IL 60611, USA. ${ }^{4}$ Department of Biomedical Engineering, University of Michigan, Ann Arbor, MI 48109, USA. ${ }^{5}$ Women's Health Research Institute, Northwestern University Feinberg School of Medicine, Chicago, IL 60611, USA. 'Department of Chemical and Biological Engineering, Northwestern University Feinberg School of Medicine, Evanston, IL 60208, USA. 


\section{Received: 3 April 2019 Accepted: 16 April 2019}

Published online: 10 June 2019

\section{References}

1. Nilsson E, Zhang B, Skinner MK. Gene bionetworks that regulate ovarian primordial follicle assembly. BMC Genomics. 2013;14:496.

2. Sugiura K, Pendola FL, Eppig JJ. Oocyte control of metabolic cooperativity between oocytes and companion granulosa cells: energy metabolism. Dev Biol. 2005;279(1):20-30

3. Eppig JJ, Pendola FL, Wigglesworth K, Pendola JK. Mouse oocytes regulate metabolic cooperativity between granulosa cells and oocytes: amino acid transport. Biol Reprod. 2005;73(2):351-7.

4. Downs SM, Verhoeven AD. Glutamine and the maintenance of meiotic arrest in mouse oocytes: influence of culture medium, glucose, and cumulus cells. Biol Reprod. 2002:66:116.

5. Downs SM, Daniel SAJ, Bornslaeger EA, Hoppe PC, Eppig JJ. Maintenance of meiotic arrest in mouse oocytes by purines - modulation of camp levels and camp phosphodiesterase activity. Gamete Res. 1989;23(3):323-34.

6. Harris SE, Gopichandran N, Picton HM, Leese HJ, Orsi NM. Nutrient concentrations in murine follicular fluid and the female reproductive tract. Theriogenology. 2005;64(4):992-1006.

7. Cinco R, Digman MA, Gratton E, Luderer U. Spatial characterization of bioenergetics and metabolism of primordial to preovulatory follicles in whole ex vivo murine ovary. Biol Reprod. 2016:95(6):129.

8. Schellenberger J, Que R, Fleming RM, Thiele I, Orth JD, Feist AM, Zielinsk DC, Bordbar A, Lewis NE, Rahmanian S, et al. Quantitative prediction of cellular metabolism with constraint-based models: the COBRA toolbox v2.0. Nat Protoc. 2011;6(9):1290-307.

9. Schellenberger J, Park JO, Conrad TM, Palsson BO. BiGG: a biochemical genetic and genomic knowledgebase of large scale metabolic reconstructions. BMC Bioinformatics. 2010:11:213.

10. Duarte NC, Becker SA, Jamshidi N, Thiele I, Mo ML, Vo TD, Srivas R, Palsson BO. Global reconstruction of the human metabolic network based on genomic and bibliomic data. Proc Natl Acad Sci U S A. 2007;104(6):1777-82.

11. Thiele I, Swainston N, Fleming RM, Hoppe A, Sahoo S, Aurich MK, Haraldsdottir H, Mo ML, Rolfsson O, Stobbe MD, et al. A community-driven global reconstruction of human metabolism. Nat Biotechnol. 2013:31(5):419-25.

12. Chandrasekaran S, Zhang J, Sun Z, Zhang L, Ross CA, Huang YC, Asara JM, Li H, Daley GQ, Collins JJ. Comprehensive mapping of pluripotent stem cell metabolism using dynamic genome-scale network modeling. Cell Rep. 2017;21(10):2965-77.

13. Uhlen M, Fagerberg L, Hallstrom BM, Lindskog C, Oksvold P, Mardinoglu A, Sivertsson A, Kampf C, Sjostedt E, Asplund A, et al. Tissue-based map of the human proteome. Science. 2015:347(6220):1260419.

14. O'Brien EJ, Monk JM, Palsson BO. Using genome-scale models to predict biological capabilities. Cell. 2015;161(5):971-87.

15. Folger O, Jerby L, Frezza C, Gottlieb E, Ruppin E, Shlomi T. Predicting selective drug targets in cancer through metabolic networks. Mol Syst Biol. 2011;7:501.

16. Heinken A, Sahoo S, Fleming RM, Thiele I. Systems-level characterization of a host-microbe metabolic symbiosis in the mammalian gut. Gut Microbes. 2013;4(1):28-40

17. Vlassis N, Pacheco MP, Sauter T. Fast reconstruction of compact contextspecific metabolic network models. PLoS Comput Biol. 2014;10(1):e1003424.

18. Pan H, O'Brien MJ, Wigglesworth K, Eppig JJ, Schultz RM. Transcript profiling during mouse oocyte development and the effect of gonadotropin priming and development in vitro. Dev Biol. 2005;286(2):493-506.

19. Wigglesworth K, Lee KB, Emori C, Sugiura K, Eppig JJ. Transcriptomic diversification of developing cumulus and mural granulosa cells in mouse ovarian follicles. Biol Reprod. 2015;92(1):23.

20. Charlier C, Montfort J, Chabrol O, Brisard D, Nguyen T, Le Cam A, RichardParpaillon L, Moreews F, Pontarotti P, Uzbekova S, et al. Oocyte-somatic cells interactions, lessons from evolution. BMC Genomics. 2012:13:560.

21. Patil KR, Nielsen J. Uncovering transcriptional regulation of metabolism by using metabolic network topology. P Natl Acad Sci USA. 2005;102(8):2685-9.

22. Rosvall M, Axelsson D, Bergstrom CT. The map equation. Eur Phys J-Spec Top. 2009;178(1):13-23

23. Rosvall M, Bergstrom CT. Multilevel compression of random walks on networks reveals hierarchical organization in large integrated systems. PLoS One. 2011:6(4):e18209.

24. Fortunato S. Community detection in graphs. Phys Rep. 2010;486(3-5):75-174.
25. Redding GP, Bronlund JE, Hart AL. Mathematical modelling of oxygen transport-limited follicle growth. Reproduction. 2007;133(6):1095-106.

26. Collado-Fernandez E, Picton HM, Dumollard R. Metabolism throughout follicle and oocyte development in mammals. Int J Dev Biol. 2012;56(10-12):799-808.

27. Ogata H, Goto S, Sato K, Fujibuchi W, Bono H, Kanehisa M. KEGG: Kyoto encyclopedia of genes and genomes. Nucleic Acids Res. 1999;27(1):29-34

28. Smith LP, Nierstenhoefer M, Yoo SW, Penzias AS, Tobiasch E, Usheva A. The bile acid synthesis pathway is present and functional in the human ovary. PLoS One. 2009;4(10):e7333.

29. Paczoskaeliasiewicz H, Rzasa J. Serotonin content in the wall of preovulatory and postovulatory follicles of the domestic hen. Folia Biol-Krakow. 1995; 43(1-2):75-9.

30. Dunning KR, Russell DL, Robker RL. Lipids and oocyte developmental competence: the role of fatty acids and beta-oxidation. Reproduction. 2014; 148(1):R15-27.

31. Roland JT, Bryant DM, Datta A, Itzen A, Mostov KE, Goldenring JR. Rab GTPase-Myo5B complexes control membrane recycling and epithelial polarization. Proc Natl Acad Sci U S A. 2011;108(7):2789-94.

32. Band V, Kharbanda SM, Murugesan K, Faroog A. Prostacyclin and steroidogenesis in goat ovarian cell-types in vitro. Prostaglandins. 1986;31(3): $509-25$

33. Kawai T, Yanaka N, Richards JS, Shimada M. De novo-synthesized retinoic acid in ovarian antral follicles enhances FSH-mediated ovarian follicular cell differentiation and female fertility. Endocrinology. 2016;157(5):2160-72.

34. Bulbul T, Akosman MS, Yilmaz O, Ulutas E, Bulbul A. Supplementary dietary nitric oxide donor (sodium nitroprusside) or inhibitor (N-G-nitro-l-arginine methyl ester) depressed growth performance and ovarian primordial and primary follicles in Japanese quail (Coturnix coturnix japonica) in a dosedependent manner. Br Poult Sci. 2015;56(1):113-20.

35. Pelland AMD, Corbett HE, Baltz JM. Amino acid transport mechanisms in mouse oocytes during growth and meiotic maturation. Biol Reprod. 2009; 81(6):1041-54

36. Downs SM, Coleman DL, Eppig JJ. Maintenance of murine oocyte meiotic arrest - uptake and metabolism of hypoxanthine and adenosine by cumulus cell-enclosed and denuded oocytes. Dev Biol. 1986;117(1):174-83.

37. Downs SM, Coleman DL, Wardbailey PF, Eppig JJ. Hypoxanthine is the principal inhibitor of murine oocyte maturation in a low-molecular weight fraction of porcine follicular-fluid. P Natl Acad Sci USA. 1985;82(2):454-8.

38. Drahorad J, Tesarik J, Cechova D, Vilim V. Proteins and glycosaminoglycans in the intercellular matrix of the human cumulus-oophorus and their effect on conversion of proacrosin to acrosin. J Reprod Fertil. 1991;93(2):253-62.

39. Tagler D, Makanji Y, Tu T, Bernabe BP, Lee R, Zhu J, Kniazeva E, Hornick JE, Woodruff TK, Shea LD. Promoting extracellular matrix remodeling via ascorbic acid enhances the survival of primary ovarian follicles encapsulated in alginate hydrogels. Biotechnol Bioeng. 2014;111(7):1417-29.

40. Su YQ, Sugiura K, Eppig JJ. Mouse oocyte control of granulosa cell development and function: paracrine regulation of cumulus cell metabolism. Semin Reprod Med. 2009;27(1):32-42.

41. Meehan TF, Conte N, West DB, Jacobsen JO, Mason J, Warren J, Chen CK, Tudose I, Relac M, Matthews P, et al. Disease model discovery from 3,328 gene knockouts by the international mouse phenotyping consortium. Nat Genet. 2017;49(8):1231-+.

42. Sinclair KD, Allegrucci C, Singh R, Gardner DS, Sebastian S, Bispham J, Thurston A, Huntley JF, Rees WD, Maloney CA, et al. DNA methylation, insulin resistance, and blood pressure in offspring determined by maternal periconceptional B vitamin and methionine status. Proc Natl Acad Sci U S A 2007;104(49):19351-6.

43. Huxtable RJ. Physiological actions of taurine. Physiol Rev. 1992;72(1):101-63.

44. Banks MA, Porter DW, Martin WG, Castranova V. Taurine protects against oxidant injury to rat alveolar pneumocytes. Adv Exp Med Biol. 1992;315: 341-54.

45. Schaffer SW, Ballard C, Azuma J. Mechanisms underlying physiological and pharmacological actions of taurine on myocardial calcium transport. Adv Exp Med Biol. 1994:359:171-80.

46. Sforza C, Vizzotto L, Ferrario VF, Forabosco A. Position of follicles in normal human ovary during definitive histogenesis. Early Hum Dev. 2003;74(1):27-35.

47. Eppig JJ, OBrien MJ. Development in vitro of mouse oocytes from primordial follicles. Biol Reprod. 1996;54(1):197-207.

48. Zheng W, Zhang H, Gorre N, Risal S, Shen Y, Liu K. Two classes of ovarian primordial follicles exhibit distinct developmental dynamics and physiological functions. Hum Mol Genet. 2014;23(4):920-8. 
49. Yuan M, Breitkopf SB, Yang X, Asara JM. A positive/negative ion-switching, targeted mass spectrometry-based metabolomics platform for bodily fluids, cells, and fresh and fixed tissue. Nat Protoc. 2012;7(5):872-81.

50. Luo X, Li L. Metabolomics of small numbers of cells: metabolomic profiling of 100, 1000, and 10000 human breast cancer cells. Anal Chem. 2017;89:11664.

51. Anastacio A, Rodriguez-Wallberg KA, Chardonnet S, Pionneau C, Federici C, Almeida Santos T, Poirot C. Protein profile of mouse ovarian follicles grown in vitro. Mol Hum Reprod. 2017;23:827.

52. Shlomi T, Cabili MN, Herrgard MJ, Palsson BO, Ruppin E. Network-based prediction of human tissue-specific metabolism. Nat Biotechnol. 2008;26(9): 1003-10.

53. Haraldsdottir HS, Thiele I, Fleming RMT. Comparative evaluation of open source software for mapping between metabolite identifiers in metabolic network reconstructions: application to Recon 2. J Cheminform. 2014;6:2.

54. Sigurdsson Ml, Jamshidi N, Steingrimsson E, Thiele I, Palsson BO. A detailed genome-wide reconstruction of mouse metabolism based on human recon 1. BMC Syst Biol. 2010;4:140.

55. Thiele I, Vlassis N, Fleming RM. fastGapFill: efficient gap filling in metabolic networks. Bioinformatics. 2014;30(17):2529-31.

56. Xu M, Kreeger PK, Shea LD, Woodruff TK. Tissue-engineered follicles produce live, fertile offspring. Tissue Eng. 2006;12(10):2739-46.

57. Skory RM, Bernabe BP, Galdones E, Broadbelt LJ, Shea LD, Woodruff TK. Microarray analysis identifies COMP as the most differentially regulated transcript throughout in vitro follicle growth. Mol Reprod Dev. 2013;80(2): $132-44$.

58. Sean D, Meltzer PS. GEOquery: a bridge between the gene expression omnibus (GEO) and BioConductor. Bioinformatics. 2007;23(14):1846-7.

59. Brazma A, Parkinson H, Sarkans U, Shojatalab M, Vilo J, Abeygunawardena N, Holloway E, Kapushesky M, Kemmeren P, Lara GG, et al. ArrayExpress - a public repository for microarray gene expression data at the EBI. Nucleic Acids Res. 2003;31(1):68-71.

60. Johnson WE, Li C, Rabinovic A. Adjusting batch effects in microarray expression data using empirical Bayes methods. Biostatistics. 2007;8(1):118-27.

61. Smyth GK. Linear models and empirical bayes methods for assessing differential expression in microarray experiments. Stat Appl Genet Mol Biol. 2004;3(1):Article3 1-25.

62. Benjamini Y, Hochberg Y. Controlling the false discovery rate - a practical and powerful approach to multiple testing. J Roy Stat Soc B Met. 1995;57(1): 289-300.

63. Pedersen T. Determination of follicle growth rate in the ovary of the immature mouse. J Reprod Fertil. 1970;21(1):81-93.

Ready to submit your research? Choose BMC and benefit from:

- fast, convenient online submission

- thorough peer review by experienced researchers in your field

- rapid publication on acceptance

- support for research data, including large and complex data types

- gold Open Access which fosters wider collaboration and increased citations

- maximum visibility for your research: over $100 \mathrm{M}$ website views per year

At $\mathrm{BMC}$, research is always in progress.

Learn more biomedcentral.com/submissions 